\title{
CYCLE AND PATH EMBEDDING ON 5-ARY N-CUBES
}

\author{
Tsong-Jie Lin ${ }^{1}$, Sun-Yuan Hsieh ${ }^{1}$ And Hui-Ling Huang ${ }^{2}$
}

\begin{abstract}
We study two topological properties of the 5-ary $n$-cube $Q_{n}^{5}$. Given two arbitrary distinct nodes $x$ and $y$ in $Q_{n}^{5}$, we prove that there exists an $x-y$ path of every length ranging from $2 n$ to $5^{n}-1$, where $n \geq 2$. Based on this result, we prove that $Q_{n}^{5}$ is 5 -edge-pancyclic by showing that every edge in $Q_{n}^{5}$ lies on a cycle of every length ranging from 5 to $5^{n}$.
\end{abstract}

Mathematics Subject Classification. 68R10,68R05, 05C12.

\section{INTRODUCTION}

One of the most important problems in interconnection networks is the embedding problem. All algorithms developed for network topology $A$ can be directly applied to topology $B$ if topology $A$ can be embedded into topology $B$. Linear arrays and rings are two fundamental networks for parallel and distributed computation. Numerous efficient algorithms based on linear arrays and rings for solving various algebraic and graph problems have been studied $[1,8,10,12]$. These applications motivated us to embed paths (linear arrays) and cycles (rings) into networks.

An interconnection network (network for short) is usually represented by a graph where vertices represent processors and edges represent communication links between processors. A graph $G=(V, E)$ is a pair comprised of the vertex set $V$ and the edge set $E$, where $V$ is a finite set and $E$ is a subset of $\{(u, v):(u, v)$ is an unordered pair of $V\}$. We sometimes use $V(G)$ and $E(G)$ to denote the vertex set and the edge set of $G$, respectively.

Keywords and phrases. Graph-theoretic interconnection networks, hypercubes, $k$-ary $n$-cubes, panconnectivity, edge-pancyclicity.

${ }^{1}$ Department of Computer Science and Information Engineering, National Cheng Kung University, No.1, University Road, Tainan 70101, Taiwan; tsong0215@yahoo.com.tw; hsiehsy@mail.ncku.edu.tw

2 Department of Information Management, Southern Taiwan University, No. 1, NanTai Street, Tainan 71005, Taiwan; hlhuang@mail.stut.edu.tw 
A graph $G=(V, E)$ is said to be panconnected (respectively, bipanconnected) if for two arbitrary distinct nodes $x$ and $y$ of $G$, there exists an $x$ - $y$ path of every length $l$ for $d(x, y) \leq l \leq|V|-1$ (respectively, $2 \mid(l-d(x, y)))^{1}$, where $d(x, y)$ is the distance of $x$ and $y$ (the length of a shortest path between $x$ and $y$ ). On the other hand, a graph $G=(V, E)$ is pancyclic (respectively, bipancyclic) if $G$ contains a cycle of every length (respectively, every even length) from three (respectively, four) to $|V|$. A bipancyclic graph $G$ is further said to be edge-bipancylic if for any edge $e$ of $G$, there exists a cycle $C$ of every even length such that $e$ is in $C$. Wang et al. [16] showed that $Q_{2}^{k}$ is both bipanconnected and bipancyclic, and $Q_{n}^{k}$ is Hamiltonian-connected when $k$ is odd.

Many networks have been studied as attractive topologies for distributed and parallel systems, including mesh, torus (also called a wrap-around mesh), hypercube, and $k$-ary $n$-cube. In fact, the $k$-ary $n$-cube is an $n$-dimensional torus with each dimension of the same size $k$, and the hypercube is a $k$-ary $n$-cube with $k=2$. Note that a mesh is a subgraph of a torus. Several parallel machines, both commercial and experimental, have been proposed based on $k$-ary $n$-cube, for example, Cray T3D and T3E (3D torus) [11], the Mosaic ( $k$-ary $n$-cube) [13], and the iWarp (torus) [2]. In particular, the $k$-ary $n$-cube has been one of the most common interconnection networks for multi-processor systems $[3,5,6,16]$.

Quite recently, Hsieh et al. [7] have investigated the panconnectivity and edgepancyclicity of 3-ary $n$-cubes $Q_{n}^{3}$. In this paper, we further investigate the panconnectivity and edge-pancyclicity of the 5 -ary $n$-cube $Q_{n}^{5}$ which is a more complicated topology than $Q_{n}^{3}$. Given two arbitrary distinct nodes $x$ and $y$ in $Q_{n}^{5}$, we prove that there exists an $x-y$ path of length $l$ ranging from $2 n$ to $5^{n}-1$, where $2 n$ is the diameter of $Q_{n}^{5}$. Based on this result, we prove that every edge in $Q_{n}^{5}$ lies on a cycle of every length ranging from 5 to $5^{n}$. The remainder of this paper is organized as follows. In the next section, some basic definitions and notations are introduced. Our main results are presented in Sections 3 and 4. Finally, some concluding remarks are presented in Section 5.

\section{Preliminaries}

Two vertices $u$ and $v$ are adjacent if $(u, v) \in E(G)$. A subgraph of $G=(V, E)$ is a graph $\left(V^{\prime}, E^{\prime}\right)$ such that $V^{\prime} \subseteq V$ and $E^{\prime} \subseteq E$. Given a set $V^{\prime} \subseteq V$, the subgraph of $G=(V, E)$ induced by $V^{\prime}$ is the graph $G^{\prime}=\left(V^{\prime}, E^{\prime}\right)$, where $E^{\prime}=\{(u, v) \in$ $\left.E: u, v \in V^{\prime}\right\}$. A path $P\left[v_{0}, v_{k}\right]=\left\langle v_{0}, v_{1}, \ldots, v_{k}\right\rangle$ in a graph $G$ is a sequence of distinct vertices such that any two consecutive vertices are adjacent, and we call $v_{0}$ and $v_{k}$ the end-vertices of the path. A cycle is a path $P\left[v_{0}, v_{k}\right]$ with $v_{0}=v_{k}$ and $k \geq 3$. A path with end-vertices $u$ and $v$ is said to be a $u-v$ path. The length of a path is the number of edges contained in the path. The distance between $u$ and $v$, denoted by $d_{G}(u, v)$, is the length of a shortest path $P[u, v]$. A path may contain another path as a subpath, denoted by $\left\langle v_{0}, v_{1}, \ldots, v_{i}, P\left[v_{i}, v_{j}\right], v_{j}, v_{j+1}, \ldots, v_{k}\right\rangle$, where

\footnotetext{
${ }^{1}$ The notation $d \mid a$ means that $a=k d$ for some integer $k$.
} 


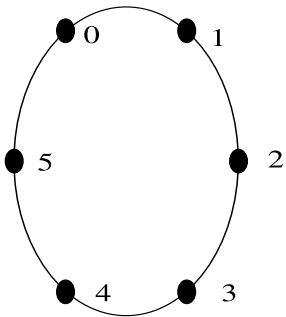

(a)

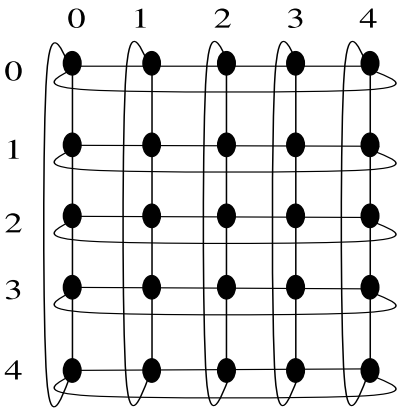

(b)

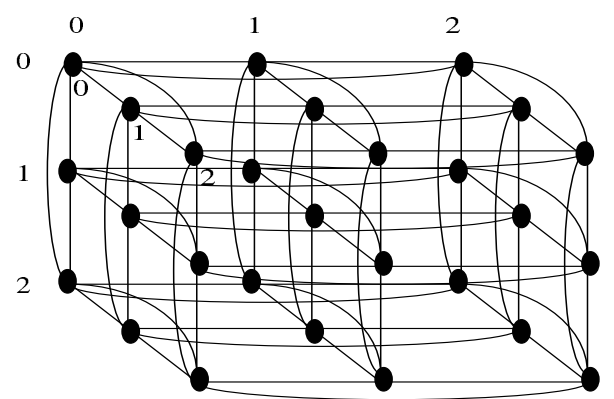

(c)

Figure 1. (a) $Q_{1}^{6}$, (b) $Q_{2}^{5}$, and (c) $Q_{3}^{3}$.

$P\left[v_{i}, v_{j}\right]=\left\langle v_{i}, v_{i+1}, \ldots, v_{j-1}, v_{j}\right\rangle$. A cycle (respectively, path) in $G$ is called a Hamiltonian cycle (respectively, Hamiltonian path) if it contains every vertex of $G$ exactly once. A graph $G$ is said to be Hamiltonian if it contains a Hamiltonian cycle, and Hamiltonian-connected if there exists a Hamiltonian path between every two distinct vertices of $G$.

A graph $G=(V, E)$ is pancyclic, if $G$ contains any cycle of length $l$ satisfying $3 \leq l \leq|V|$, that is, any cycle of length $l$ can be embedded into $G$ with dilation one. Furthermore, $G$ is edge-pancyclic if every edge of $G$ lies on a cycle of every length from 3 to $|V|$. $G$ is L-edge-pancyclic if every edge of $G$ lies on a cycle of every length from $L$ to $|V|$. The $k$-ary $n$-cube $Q_{n}^{k}(k \geq 2$ and $n \geq 1)$ has $N=k^{n}$ nodes each of the form $x=x_{n} x_{n-1} \ldots x_{1}$, where $0 \leq x_{i}<k$ for all $1 \leq i \leq n$. Two nodes $x=x_{n} x_{n-1} \ldots x_{1}$ and $y=y_{n} y_{n-1} \ldots y_{1}$ in $Q_{n}^{k}$ are adjacent if and only if there exists an integer $j, 1 \leq j \leq n$, such that $x_{j}=y_{j} \pm 1(\bmod k)$ and $x_{l}=y_{l}$ for every $l \in\{1,2, \ldots, n\}-\{j\}$. For clarity of presentation, we omit writing " $(\bmod k)$ " in similar expressions for the remainder of the paper. Note that each node has degree $2 n$ when $k \geq 3$, and $n$ when $k=2$. Obviously, $Q_{1}^{k}$ is a cycle of length $k$, $Q_{n}^{2}$ is an $n$-dimensional hypercube, and $Q_{2}^{k}$ is a $k \times k$ wrap-around mesh. In this paper, we pay our attention on $k=5$. Figure 1 illustrates $Q_{1}^{6}, Q_{2}^{5}$, and $Q_{3}^{3}$. 
The $i$ th position, from the right to the left, of the $n$-bit string $x_{n} x_{n-1} \ldots x_{1}$ is called the $i$-dimension. We can partition $Q_{n}^{5}$ along the $i$-dimension by regarding the graph comprised of 5 disjoint copies, $Q_{n-1}^{5}[0], Q_{n-1}^{5}[1], Q_{n-1}^{5}[2], Q_{n-1}^{5}[3]$, and $Q_{n-1}^{5}[4],{ }^{2}$ where $Q_{n-1}^{5}[j]$ is the subgraph of $Q_{n}^{5}$ induced by $\left\{x \in V\left(Q_{n}^{5}\right) \mid\right.$ the $i$ th bit $x_{i}$ of $x=x_{n} x_{n-1} \ldots x_{1}$ is fixed by $\left.j \in\{0,1,2,3,4\}\right\}$. We call each $Q_{n-1}^{5}[j]$ a subcube of $Q_{n}^{5}$. Note that $Q_{n-1}^{5}[j]$ is isomorphic to a 5 -ary $(n-1)$-cube. Clearly, there are exactly $5^{n-1}$ edges which form a perfect matching between $Q_{n-1}^{5}[j]$ and $Q_{n-1}^{5}[j+1]$. We call $Q_{n-1}^{5}[j]$ and $Q_{n-1}^{5}[j+1]$ adjacent subcubes, and call the edges between two adjacent subcubes bridges.

\section{Panconnectivity of 5 -ARy $n$-Cubes}

In this section, we investigate the panconnectivity of 5-ary $n$-cubes. We first provide some previously known properties which are useful in our method.

Lemma 1 [3]. The diameter of $Q_{n}^{k}$ equals $\left\lfloor\frac{k}{2}\right\rfloor n$.

Lemma 2 [16]. The following two statements hold:

(1) $Q_{2}^{k}$ is bipanconnected.

(2) $Q_{n}^{k}$ is Hamiltonian-connected when $k$ is odd.

Lemma 3. For any two distinct nodes $x, y \in V\left(Q_{2}^{5}\right)$ and any integer $l$ with $4 \leq$ $l \leq 24, Q_{2}^{5}$ contains an $x-y$ path of length $l$.

Proof. We attempt to construct an $x-y$ path of every length $l$ for $4 \leq l \leq 24$. Due to the structure property of $Q_{2}^{5}$, we need to consider the following five cases.

Case 1. $x=00$ and $y=01$.

Case 1.1: $l$ is odd. We have $d(x, y)=1$ and since $Q_{2}^{k}$ is bipanconnected by Lemma 2(1), all desired $x-y$ paths can be obtained.

Case 1.2: $l$ is even. A desired path of every even length can be obtained from a Hamiltonian path $\langle 00,04,14,24,34,44,43,42,41,40$, $30,31,32,33,23,22,21,20,10,11,12,13,03,02,01\rangle$ by subtracting length 2 successively till length of 4 , using a routing strategy illustrated in Figures $2 \mathrm{a}-2 \mathrm{f}$.

Case 2. $x=00$ and $y=02$.

Case 2.1: $l$ is odd. A desired path of every odd length can be obtained from a path $\langle 00,04,14,24,34,44,43,42,41,40,30,31,32,33,23,22$,

$21,20,10,11,12,13,03,02\rangle$ by subtracting length 2 successively till length of 5 , using a routing strategy illustrated in Figures $3 \mathrm{a}-3 \mathrm{f}$.

Case 2.2: $l$ is even. We have $d(x, y)=2$ and since $Q_{2}^{k}$ is bipanconnected by Lemma 2(1), all desired $x-y$ paths can be obtained.

\footnotetext{
${ }^{2}$ Since the index $i$ is not necessary to be specified, we omit it from the notation.
} 


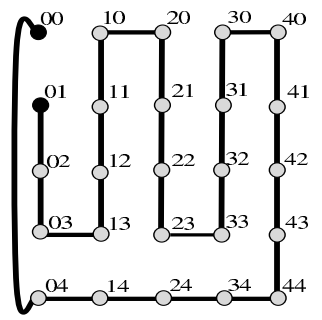

(a)

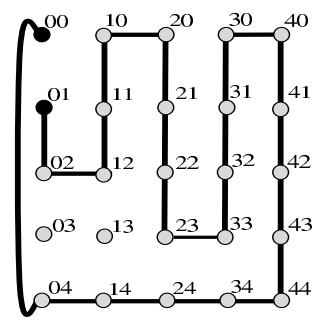

(b)

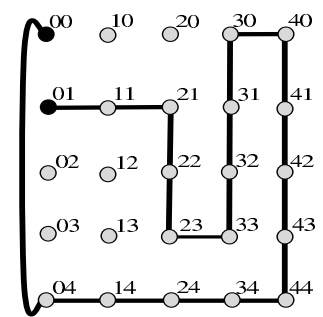

(c)

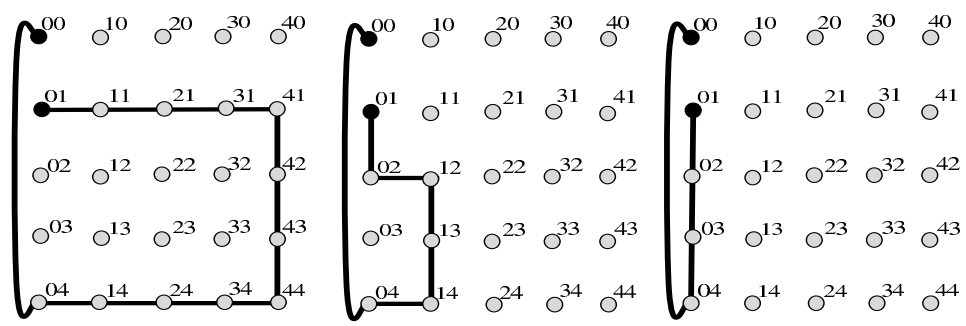

(d)

Figure 2. An illustration of case 1 in the proof of Lemma 3.

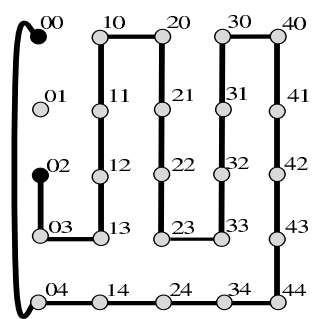

(a)

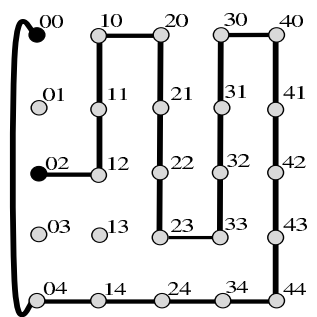

(b)

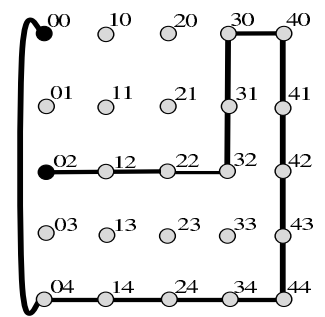

(c)

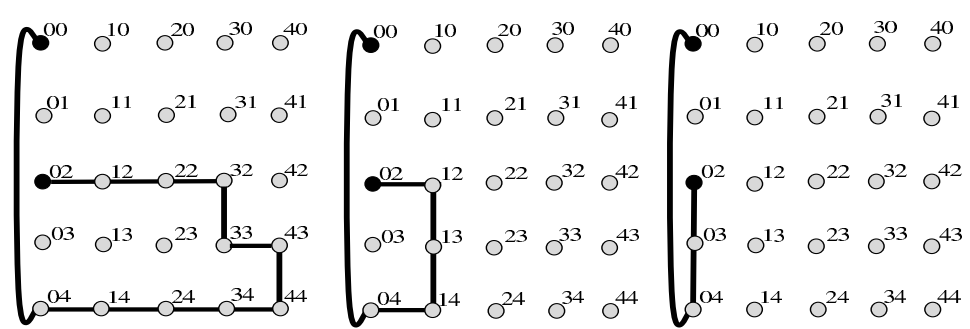

(d)

(e)

(f)

Figure 3. An illustration of case 2 in the proof of Lemma 3. 


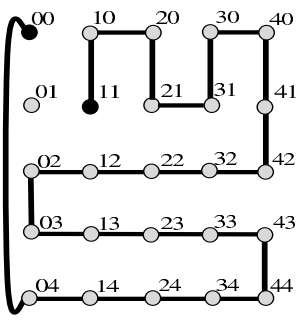

(a)

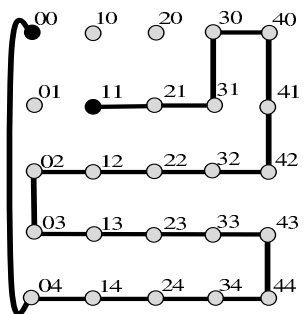

(b)

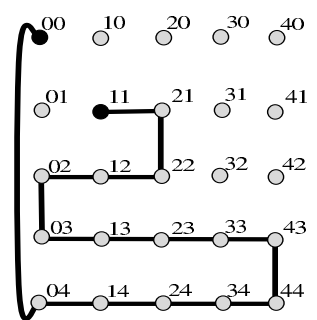

(c)

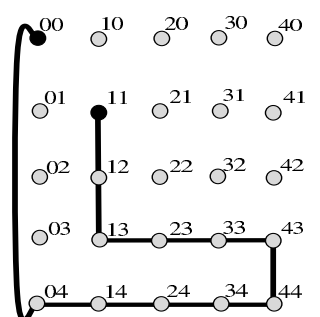

(d)

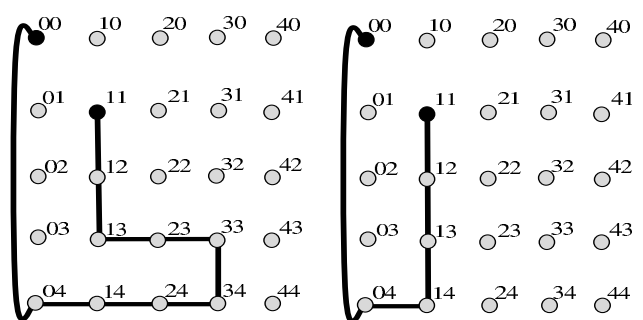

(e)

(f)

Figure 4. An illustration of case 3 in the proof of Lemma 3.

Case 3. $x=00$ and $y=11$. The proof is similar to Case 2 (see Fig. 4).

Case 4. $x=00$ and $y=12$. The proof is similar to Case 1 (see Fig. 5).

Case 5. $x=00$ and $y=22$. The proof is similar to Case 2 (see Fig. 6).

For convenience, we use the notation " $u \mapsto v$ " to mean that $(u, v)$ is a bridge between two adjacent subcubes.

Theorem 1. For any two distinct nodes $x, y \in V\left(Q_{n}^{5}\right)$, where $n \geq 2$, and any integer $l$ with $2 n \leq l \leq 5^{n}-1$, there exists an $x$-y path of length $l$.

Proof. We prove this theorem by induction on $n$. By Lemma 3, the base result holds for $n=2$. Suppose that the result holds for the 5 -ary $(n-1)$-cube. We now consider $Q_{n}^{5}$, where $n \geq 3$. We partition $Q_{n}^{5}$ along the dimension $i$ into five subcubes $Q_{n-1}^{5}[0], Q_{n-1}^{5}[1], Q_{n-1}^{5}[2], Q_{n-1}^{5}[3]$, and $Q_{n-1}^{5}[4]$. We will attempt to construct an $x-y$ path of every length $l$ with $2 n \leq l \leq 5^{n}-1$. There are the following two scenarios.

Case 1: $2 n \leq l \leq 5^{n-1}-1$. Without loss of generality, we assume that $x$ is in $Q_{n-1}^{5}[0]$. Due to the structure of $Q_{n}^{5}$, we only consider that $y$ is in $Q_{n-1}^{5}[j]$, where $j=0,1$, or 2 . Let $u_{1}$ be a node in $Q_{n-1}^{5}[1]$ such that $x \mapsto u_{1}$ if $y$ is in $Q_{n-1}^{5}[1]$. Let $u_{2}$ be a node in $Q_{n-1}^{5}[2]$ such that $x \mapsto u_{1} \mapsto u_{2}$ if $y$ is in $Q_{n-1}^{5}[2]$, where $u_{1}$ is a node in $Q_{n-1}^{5}[1]$. Let $u_{0}=x$ if $y$ is in $Q_{n-1}^{5}$ [0]. If $u_{1}=y$ or $u_{2}=y$, then we can partition $Q_{n}^{5}$ along another dimension $i^{\prime}(\neq i)$ such that $x$ and $y$ are in the same subcube. By the induction hypothesis, 


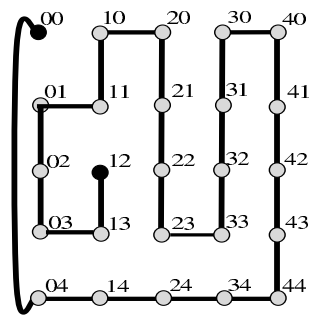

(a)

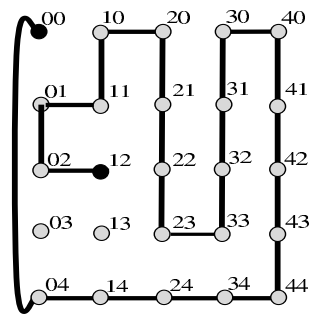

(b)

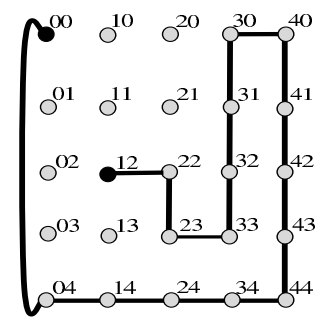

(c)

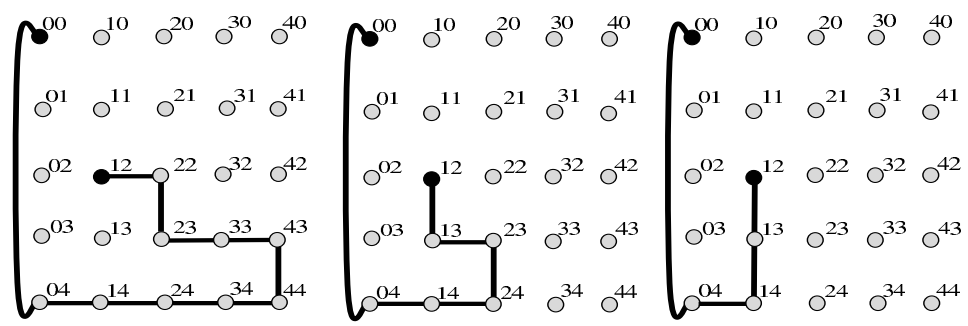

(d)

(f)

Figure 5. An illustration of case 4 in the proof of Lemma 3.

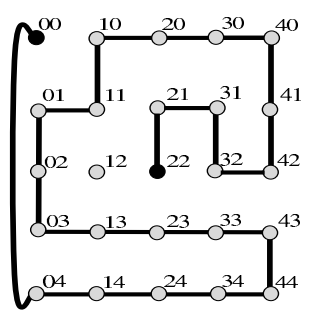

(a)

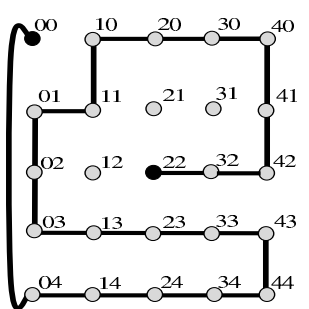

(b)

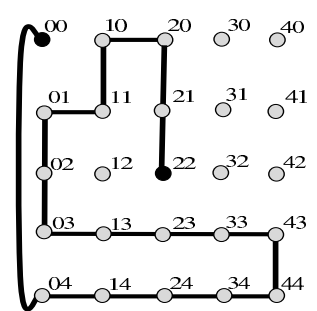

(c)

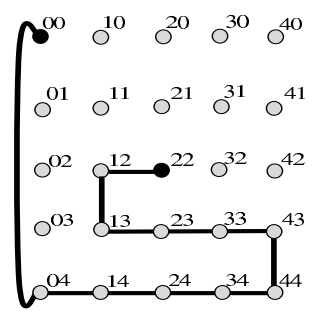

(d)

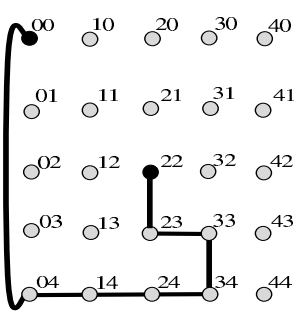

(e)

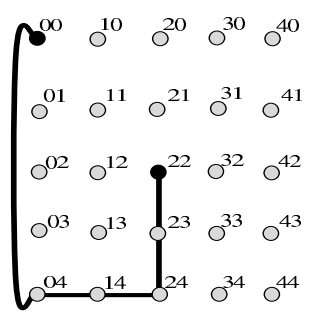

(f)

Figure 6. An illustration of case 5 in the proof of Lemma 3. 
$Q_{n-1}^{5}[j]$ contains a path $P_{j}\left[u_{j}, y\right]$ of length $l_{j}$ with $2(n-1) \leq l_{j} \leq 5^{n-1}-1$. An $x-y$ path of length $l_{1}$ with $2(n-1)+1 \leq l_{1} \leq 5^{n-1}$ can be constructed by $\left\langle x, u_{1}, P_{1}\left[u_{1}, y\right], y\right\rangle$ if $y$ is in $Q_{n-1}^{5}$ [1], and an $x$-y path of length $l_{2}$ with $2 n \leq l_{2} \leq 5^{n-1}+1$ can be constructed by $\left\langle x, u_{1}, u_{2}, P_{2}\left[u_{2}, y\right], y\right\rangle$ if $y$ is in $Q_{n-1}^{5}[2]$. Therefore, any path of the specified length can be constructed.

Case 2: $5^{n-1} \leq l \leq 5^{n}-1$. Without loss of generality, we assume that $x$ is in $Q_{n-1}^{5}[0]$. Due to the structure of $Q_{n}^{5}$, we only consider $y$ to be in $Q_{n-1}^{5}[0], Q_{n-1}^{5}[1]$, or $Q_{n-1}^{5}[2]$. No matter which subcube $y$ is in, we can construct an $x-y$ path by using a routing strategy illustrated in Figure 7. Note that $P_{0}$ is an $x-y$ path in Figure 7a and an $x-v$ path in Figure $7 \mathrm{~b}$ and c. By the induction hypothesis, $Q_{n-1}^{5}[k]$ contains a path $P_{k}$ of length $l_{k}$ with $2(n-1) \leq l_{k} \leq 5^{n-1}-1$, where $0 \leq k \leq 4$. Thus, an $x-y$ path of length $l=l_{0}+l_{1}+l_{2}+l_{3}+l_{4}+4$ with $10 n-6 \leq 5^{n-1} \leq l \leq 5^{n}-1$ can be constructed, where $n \geq 3$.

The proof is completed from the above two cases.

Remarks 1. Note that when $d(x, y)=1$, there is no $x-y$ path of length 2 in $Q_{n}^{5}$. Therefore, the range of length $l$ in the statement of Theorem 1 cannot start with $d(x, y)$.

\section{EDGE-PANCYCLICITY OF 5-ARY $n$-CUBES}

In this section, we investigate the edge-pancyclicity of 5-ary $n$-cubes according to the results obtained in Section 3.

Lemma 4. For any edge $(x, y) \in E\left(Q_{2}^{5}\right)$ and any integer $l$ with $5 \leq l \leq 25$, there exists a cycle $C$ of length $l$ such that $(x, y)$ is in $C$.

Proof. Given two adjacent nodes $x$ and $y$ in $Q_{2}^{5}$, without loss of generality, let $x=00$ and $y=01$, there exists an $x-y$ path $P[x, y]$ of every length $l$ for $4 \leq l \leq 24$ by the Case 1 of proof in Lemma 3. Then $P[x, y]+(x, y)$ forms a cycle of every length $l$ for $5 \leq l \leq 25$. Therefore, the result holds.

Theorem 2. For any edge $(x, y) \in E\left(Q_{n}^{5}\right)$, where $n \geq 1$, and any integer $l$ with $5 \leq l \leq 5^{n}$, there exists a cycle $C$ of length $l$ such that $(x, y)$ is in $C$. That is, $Q_{n}^{5}$ is 5-edge-pancyclic.

Proof. We prove this theorem by induction on $n$. The result clearly holds for $n=1$ because $Q_{1}^{5}$ is a cycle of length 5 , and also holds for $n=2$ by Lemma 4 . Suppose that the result holds for 5 -ary $(n-1)$-cubes. We now consider $Q_{n}^{5}$. We partition $Q_{n}^{5}$ along the dimension $i$ into five subcubes $Q_{n-1}^{5}[0], Q_{n-1}^{5}[1], Q_{n-1}^{5}[2], Q_{n-1}^{5}[3]$, and $Q_{n-1}^{5}$ [4]. Without loss of generality, we assume that $(x, y)$ is in $Q_{n-1}^{5}$ [0].

Case $15 \leq l \leq 5^{n-1}$ (see Fig. 8a). By the induction hypothesis, $Q_{n-1}^{5}[0]$ contains a cycle $C_{0}$ of length $l$ such that $(x, y)$ is in $C_{0}$. 


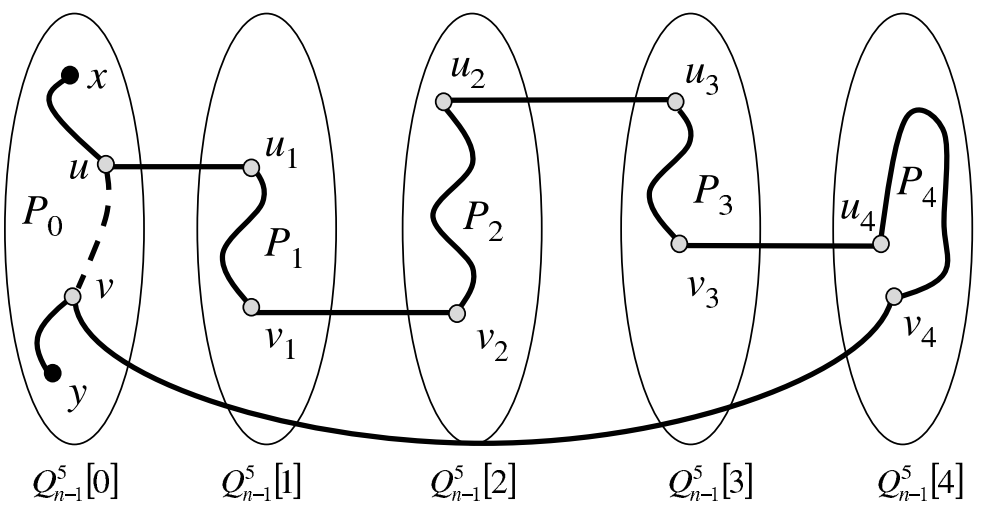

(a)
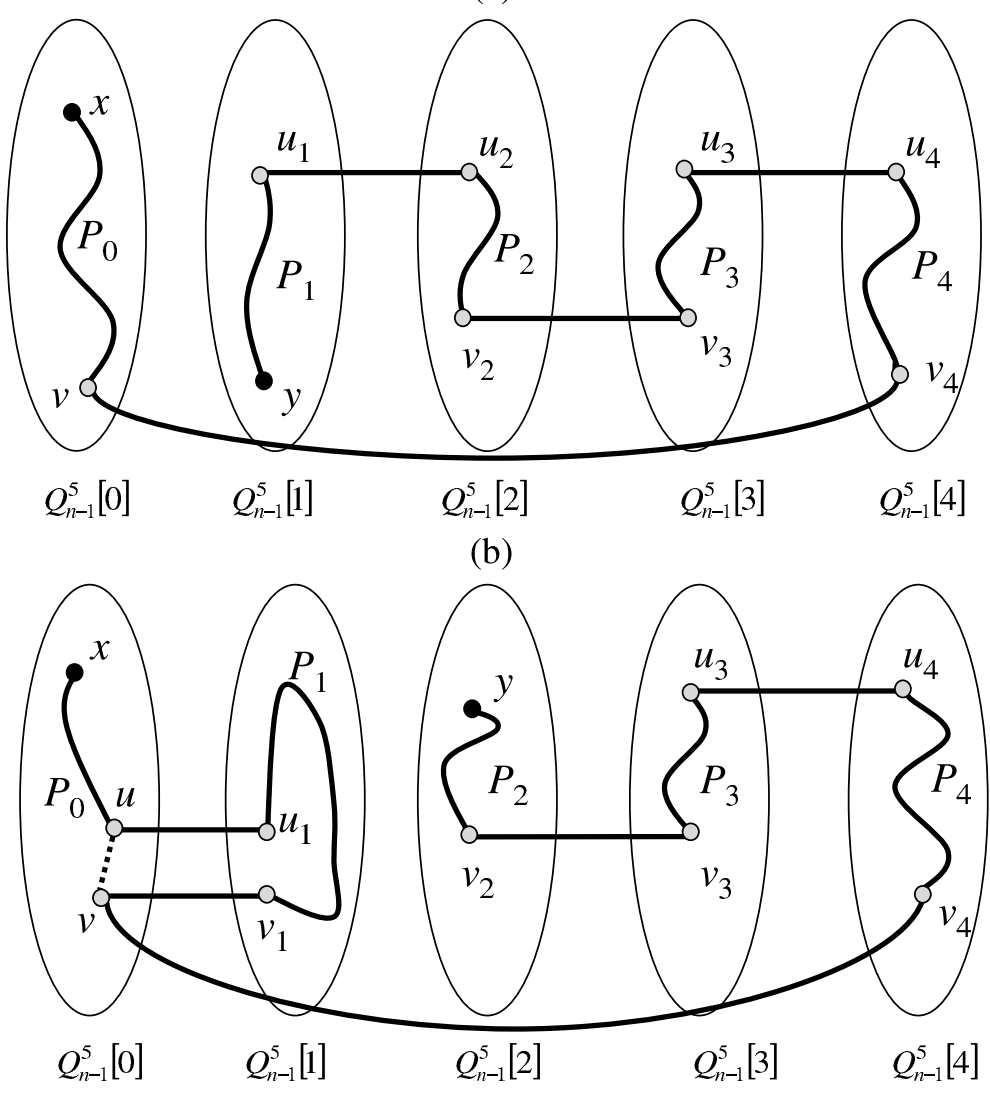

(c)

FiguRE 7. An illustration of the proof of Theorem 1. 

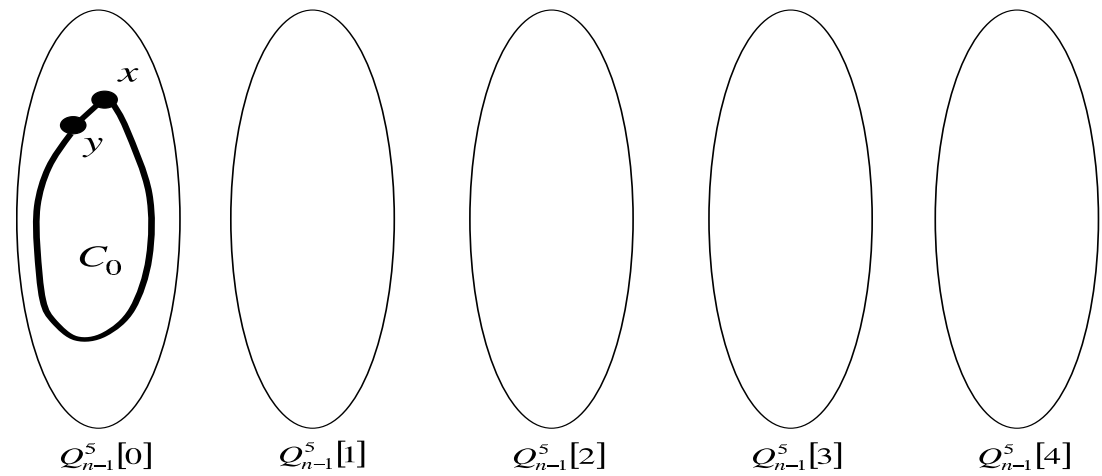

(a)

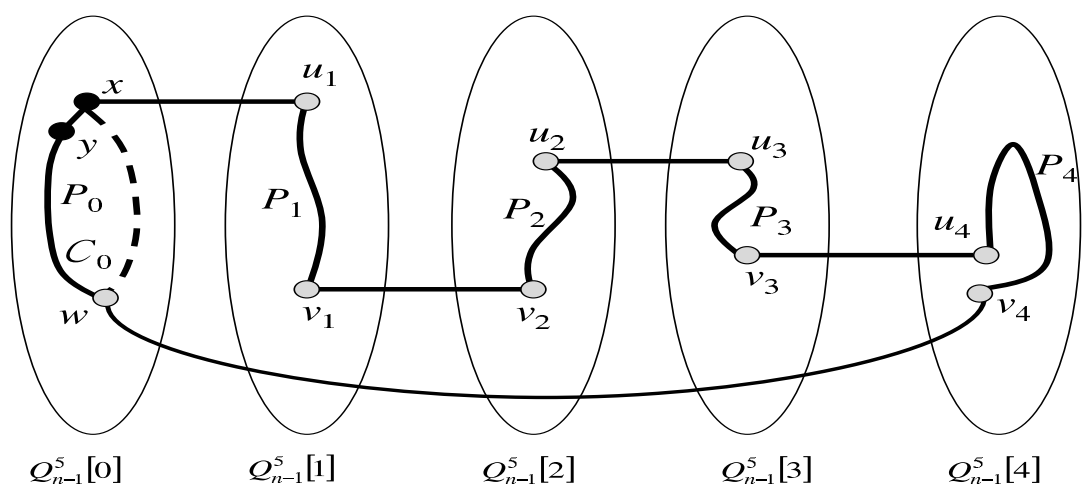

(b)

FiguRE 8. An illustration of the proof of Theorem 2.

Case $25^{n-1}+1 \leq l \leq 5^{n}$ (see Fig. 8b). By the induction hypothesis, $Q_{n-1}^{5}[0]$ contains a cycle $C_{0}$ of length $5^{n-1}$ such that $(x, y)$ is in $C_{0}$. Clearly, we can select a path $P_{0}[x, w]=\langle x, y, \ldots, w\rangle$ from $C_{0}$ whose length $l_{0}$ satisfies $5^{n-1}-8 n+4 \leq l_{0} \leq 5^{n-1}-1$. We can represent $P_{0}[x, w]$ as $\left\langle x, y, P_{0}^{\prime}[y, w], w\right\rangle$. Furthermore, we can select two distinct nodes $u_{i}$ and $v_{i}$ in $Q_{n-1}^{5}[i]$ for $i \in\{1,2,3,4\}$ such that $x \mapsto u_{1}, v_{1} \mapsto v_{2}, u_{2} \mapsto u_{3}$, $v_{3} \mapsto u_{4}$, and $v_{4} \mapsto w$. By Theorem $1, Q_{n-1}^{5}[i]$ contains a path $P_{i}\left[u_{i}, v_{i}\right]$ of length $l_{i}$ with $2(n-1) \leq l_{i} \leq 5^{n-1}-1$ for $i \in\{1,2,3,4\}$.

Therefore, $\left\langle x, y, P_{0}^{\prime}[y, w], w, v_{4}, P_{4}\left[v_{4}, u_{4}\right], u_{4}, v_{3}, P_{3}\left[v_{3}, u_{3}\right], u_{3}, u_{2}, P_{2}\left[u_{2}\right.\right.$, $\left.\left.v_{2}\right], v_{2}, v_{1}, P_{1}\left[v_{1}, u_{1}\right], u_{1}, x\right\rangle$ forms a cycle of length $l\left(=l_{0}+l_{1}+l_{2}+l_{3}+l_{4}+5\right)$ such that $(x, y)$ is in the cycle and $5^{n-1}+1 \leq l \leq 5^{n}$.

By combining cases 1-2, we complete the proof.

Remarks 2. Since $Q_{1}^{5}$ has no cycle of length $l=3,4$ and $Q_{2}^{5}$ has no cycle of length 3 , we can conclude that $Q_{n}^{5}$ for $n \geq 1$ is only 5 -edge-pancyclic. 
A graph $G$ is $L$-node-pancyclic if every node of $G$ lies on a cycle of every length from $L$ to $|V(G)|$ for some constant $L$. The following result follows directly from Theorem 2 .

Corollary 1. The 5-ary n-cube $Q_{n}^{5}$ is 5-node-pancyclic.

\section{Concluding REMarks}

In this paper, we have focused on topology embedding, where a 5 -ary $n$-cube $Q_{n}^{5}$ acts as the host graph and paths (cycles) represent the guest graphs. Given two arbitrary distinct nodes $x$ and $y$, we prove that $Q_{n}^{5}$ can embed an $x-y$ path of length $l$ ranging from $2 n$ to $5^{n}-1$, where $2 n$ is the diameter of $Q_{n}^{5}$. Based on this result, we also prove that every edge in $Q_{n}^{5}$ lies on a cycle of every length ranging from 5 to $5^{n}$.

Paths (linear arrays) and cycles (rings) are two fundamental networks for parallel and distributed computation, and are suitable for designing simple algorithms with low communication costs. Our results show that algorithms designed for paths (cycles) can also be executed well on $Q_{n}^{5}$. A future work is to extend our result to the $k$-ary $n$-cube for a general $k$.

\section{REFERENCES}

[1] S.G. Akl, Parallel Computation: Models and Methods Prentice Hall, NJ (1997).

[2] S. Borkar, R. Cohn, G. Cox, S. Gleason, T. Gross, H.T. Kung, M. Lam, B. Moore, C. Peterson, J. Pieper, L. Rankin, P.S. Tseng, J. Sutton, J. Urbanski and J. Webb, iWarp: an integrated solution to high-speed parallel computing, Proceedings of the 1988 ACM/IEEE conference on Supercomputing (1988) 330-339.

[3] B. Bose, B. Broeg, Y.G. Kwon and Y. Ashir, Lee distance and topological properties of k-ary n-cubes. IEEE Trans. Comput. 44 (1995) 1021-1030.

[4] J. Chang, J. Yang and Y. Chang, Panconnectivity, fault-Tolorant Hamiltonicity and Hamiltonian-connectivity in alternating group graphs. Networks 44 (2004) 302-310.

[5] K. Day and A.E. Al-Ayyoub, Fault diameter of k-ary n-cube Networks. IEEE Transactions on Parallel and Distributed Systems, 8 (1997) 903-907.

[6] S.A. Ghozati and H.C. Wasserman, The $k$-ary $n$-cube network: modeling, topological properties and routing strategies. Comput. Electr. Eng. (2003) 1271-1284.

[7] S.Y. Hsieh, T.J. Lin and H.-L. Huang, Panconnectivity and edge-pancyclicity of 3-ary Ncubes. J. Supercomputing 42 (2007) 225-233.

[8] F.T. Leighton, Introduction to Parallel Algorithms and Architecture: Arrays - Trees . Hypercubes. Morgan Kaufmann, San Mateo, CA (1992).

[9] M. Ma and J. M. Xu, Panconnectivity of locally twisted cubes. Appl. Math. Lett. 19 (2006) 673-677.

[10] B. Monien and H. Sudborough, Embedding one interconnection network in another. Computing Suppl. 7 (1990) 257-282.

[11] W. Oed, Massively parallel processor system CRAY T3D. Technical Report, Cray Research GmbH (1993).

[12] A.L. Rosenberg, Cycles in Networks. Technical Report: UM-CS-1991-020, University of Massachusetts, Amherst, MA, USA (1991). 
[13] C.L. Seitz et al., Submicron systems architecture project semi-annual technical report. Technical Report Caltec-CS-TR-88-18, California Institute of Technology (1988).

[14] Y. Sheng, F. Tian and B. Wei, Panconnectivity of locally connected claw-free graphs. Discrete Mathematics 203 (1999) 253-260.

[15] Z.M. Song and Y.S. Qin, A new sufficient condition for panconnected graphs. Ars Combinatoria 34 (1992) 161-166.

[16] D. Wang, T. An, M. Pan, K. Wang and S. Qu, Hamiltonian-like properies of $k$-Ary $n$ Cubes, in Proceedings PDCAT05 of Sixth International Conference on Parallel and Distributed Computing, Applications and Technologies (PDCAT05), IEEE Computer Society Press (2005) pp. 1002-1007.

Communicated by J. Hromkovic.

Received May 3, 2007. Accepted January 22, 2008. 